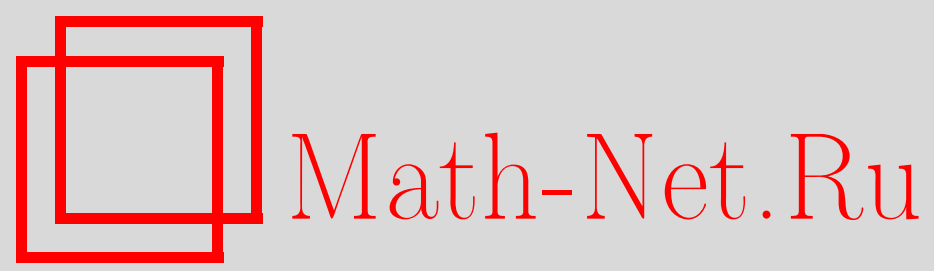

Г. И. Арончик, Метод итерационного расщепления в процедурах численного моделирования излучающих систем, Вестн. Сам. гос. техн. ун-та. Сер. Физ.мат. науки, 1998, выпуск 6, 66-77

DOI: https://doi.org/10.14498/vsgtu9

Использование Общероссийского математического портала Math-Net.Ru подразумевает, что вы прочитали и согласны с пользовательским соглашением

http://www . mathnet.ru/rus/agreement

Параметры загрузки:

IP : 35.173 .137 .237

26 апреля 2023 г., 10:21:16 


\title{
Теплофизика
}

\author{
УДК 519.6:536.3
}

\section{Г.И. Арончик}

\section{МЕТОД ИТЕРАЦИОННОГО РАСЩЕПЛЕНИЯ В ПРОЦЕДУРАХ ЧИСЛЕННОГО МОДЕЛИРОВАНИЯ ИЗЛУЧАЮЩИХ СИСТЕМ}

\begin{abstract}
Рассмотрены основные особенности постановки задач сложного теплообмена в излучающих системах. Описана структура метода итерационного расщепления, позволяющего снизить объем вычислительных затрат при математическом моделировании излучающих систем за счет использования эффективных специализированных методов решения частных задач теплопереноса. Приведены результаты построения достаточных условий сходимости алгоритма. Приведен пример реализации метода итеращионного расщепления, иллюстрирующий эффективность предложенного подхода.
\end{abstract}

Специфика методов математического моделирования излучающих систем определяется, в основном, особенностями математических моделей и алгоритмов расчета процессов радиационного теплообмена по сравнению с иными видами теплопереноса. Различный физический характер и форма описания процессов радиационного и конвективно-кондуктивного теплопереноса являются источниками существенных трудностей при разработке алгоритмов и программных средств численного моделирования такого рода систем и, в конечном итоге, при разработке алгоритмов оптимизации и управления соответствующими процессами. Перспективным направлением развития методов математического моделирования излучающих систем? на наш взгляд, является последовательное применение идеи итерационного расщепления задач сложного энергообмена. Такие алгоритмы позволяют существенно снизить объем вычислительных затрат при одновременном повышении гибкости структуры программного обеспечения, однако требуют применения специальных средств, обеспечивающих сходимость итерационных процессов. Общая схема метода итерационного расщепления описана в ряде работ автора $[1,2]$. Однако обоснование метода в ранее опубликованных работах не приведено.

Для изложения структуры метода итерационного расщепления и построения условий сходимости итераций рассмотрим постановку задачи сложного теплообмена в излучающей системе и определим структуру математической модели при наличии ряда упрощающих предположений. Если предположить, что теплофизические характеристики элементов системы не зависят от температуры, отражение и рассеяние изотропны, математическая модель сложного теплообмена в некоторой пространственной области $V$, ограниченной поверхностью $F$ может быть представлена в следующей форме:

$$
D T=\eta_{\text {погл }}(T)+\eta_{\text {погл }}\left(T_{w}\right)-\eta_{c}(T), \quad T_{\mid \tau=0}=T^{0}, T_{\mid F}=T_{w},
$$

где $\mathrm{D}$ - дифференциальный оператор уравнения энергии; $\eta_{\text {погл }}(T), \eta_{\text {погл }}\left(T_{w}\right)$ - составляющие объемной плотности потока поглощенного излучения, обусловленные собственным излучением элементов объема и поверхности соответственно; $\eta_{c}(T)$ - объемная плотность потока собственного излучения; $T_{w}$ - температура граничных поверхностей $F ; T^{0}$ - начальное распределение температур.

При принятых допущениях оператор $D$ имеет следующую структуру [3]:

$$
D=c_{p} \rho \frac{\partial}{\partial \tau}+\operatorname{div}\left(c_{p} \rho T \cdot \vec{v}\right)-\operatorname{div}(\lambda \nabla),
$$


где $c_{p}, \rho$ - теплоемкость и плотность среды; $\lambda$ - коэффициент теплопроводности; $\tau$ - время; $\vec{v}$ - вектор скорости; $\nabla$ - оператор Гамильтона.

Источник в правой части соотношения (2) представляет собой объемную плотность потока результирующего излучения, которая при заданных температурных полях в объеме $V$ и на поверхности $F$ определяется путем решения системы интегральных уравнений радиационного теплообмена [4] и может быть записана в виде

$$
\begin{gathered}
\eta_{\text {pes }}(M)=\eta_{\text {nогл }}(T)+\eta_{\text {nогл }}\left(T_{w}\right)-\eta_{c}(T)= \\
=\int_{v=0}^{\infty}\left[\begin{array}{l}
\alpha_{v}^{\prime}(M) \int_{V} 4 \alpha_{v}^{\prime}\left(M_{1}\right) E_{T, v}\left(T\left(M_{1}\right)\right) \Gamma_{V V, v}\left(M, M_{1}\right) d V_{M_{1}}+ \\
+\alpha_{v}^{\prime}(M) \int_{F} \varepsilon_{v}\left(N_{1}\right) E_{T, v}\left(T_{w}\left(N_{1}\right)\right) \Gamma_{F V, v}\left(M, N_{1}\right) d F_{N_{1}}- \\
-4 \alpha_{v}^{\prime}(M) E_{T, v}\left(T\left(M_{1}\right)\right)
\end{array}\right] d v,
\end{gathered}
$$

где $M, M_{1}, N_{1}$ - точки в объеме $V$ и на поверхности $F$ соответственно ( $\left.M, M_{1} \in V, \quad N_{1} \in F\right) ; v$ - частота излучения; $\alpha_{v}^{\prime}$ - эффективный спектральный коэффициент поглощения среды; $\varepsilon_{v}$ - спектральная степень черноты поверхности $F ; \Gamma_{V V, v}\left(M, M_{1}\right)$, $\Gamma_{F V, v}\left(M, N_{1}\right)$ - резольвенты ядер интегральных уравнений радиационного теплообмена [4].

Из соотношения (3) видно, что составляющие $\eta_{\text {nогл }}(T), \eta_{\text {nогл }}\left(T_{w}\right), \eta_{c}(T)$ соответственно равны

$$
\begin{aligned}
& \eta_{\text {nогл }}(T)=\int_{v=0}^{\infty}\left[\alpha_{v}^{\prime}(M) \int_{V} 4 \alpha_{v}^{\prime}\left(M_{1}\right) E_{T, v}\left(T\left(M_{1}\right)\right) \Gamma_{V V, v}\left(M, M_{1}\right) d V_{M_{1}}\right] d v, \\
& \eta_{\text {nогл }}\left(T_{w}\right)=\int_{v=0}^{\infty}\left[\alpha_{v}^{\prime}(M) \int_{F} \varepsilon_{v}\left(N_{1}\right) E_{T, v}\left(T_{w}\left(N_{1}\right)\right) \Gamma_{F V, v}\left(M, N_{1}\right) d F_{N_{1}}\right] d v,
\end{aligned}
$$

$$
\eta_{c}(T)=\int_{v=0}^{\infty}\left[4 \alpha_{v}^{\prime}(M) E_{T, v}(T(M))\right] d v .
$$

Доопределим задачу (1), ограничив область определения соответствующих операторов. Пусть коэффициенты в (2) достаточно гладкие. Процесс теплообмена рассматривается во временном интервале $V_{\tau}$. Тогда в рамках классической постановки краевых задач естественная область определения оператора D может быть задана в виде

$$
R(D)=\left\{T \mid T \in C^{2}(V) \times C^{1}\left(V_{\tau}\right)\right\} .
$$

Интегральные уравнения радиационного теплообмена являются интегральными уравнениями Фредгольма второго рода и имеют единственное решение по крайней мере в пространстве функций с интегрируемым квадратом $L_{2}(V)[5]$. Следовательно, естественно определить операторы $\eta_{\text {nогл }}, \eta_{c}$ как операторы в

$$
R\left(\eta_{\text {nогл }}\right)=\left\{T \mid T \in L_{2}(V) \times C^{1}\left(V_{\tau}\right)\right\}, R\left(\eta_{c}\right)=\left\{T \mid T \in L_{2}(V) \times C^{1}\left(V_{\tau}\right)\right\} .
$$

При этом время $\tau$ в интегральных уравнениях радиационного теплообмена играет роль параметра.

Так как очевидно, что $R(D) \subset R\left(\eta_{\text {погл }}\right) \subset R\left(\eta_{c}\right)$, то в качестве области определения задачи примем пространство функций, определенное в (7).

Приведенное описание задачи сложного теплообмена не является единственно возможным как с точки зрения формулировки задачи (1), так и в плане выбора конкретных функциональных пространств. Однако оно, по крайней мере, является достаточным для построения итерационного алгоритма решения задач сложного теплообмена, изложенного ниже.

В ряде практически важных случаев необходимо учитывать взаимное влияние гидродинамических и тепловых процессов, что, в первую очередь, связано с существенной зависимостью теплофизических характеристик от температуры и давления. При этом поля скорости и темпе- 
ратуры необходимо определять совместно путем решения полной системы уравнений сложного теплообмена [3]. Многие процессы в энергетических и технологических установках близки к изобарным. Поэтому наиболее существенной представляется зависимость теплофизических характеристик от температуры. Пусть, соответственно, в дифференциальном операторе $D$ величины $c_{p} \rho$ и $\lambda$ являются функциями температуры, т.е. $c_{p} \rho=c_{p} \rho(T), \lambda=\lambda(T)$. При заданном температурном поле Т поле скорости $\vec{v}$ определяется путем решения полной нелинейной системы уравнений гидродинамики. Поэтому $\vec{v}$ можно считать нелинейным оператором и записать $\vec{v}=\vec{v}(T)$. Тогда математическую модель сложного теплообмена можно представить в форме (1), где $D$ - нелинейный оператор:

$$
D T=c_{p} \rho(T) \frac{\partial T}{\partial \tau}+\operatorname{div}\left(c_{p} \rho(T) T \cdot \vec{v}(T)\right)-\operatorname{div}(\lambda(T) \nabla T)
$$

В некоторых случаях недопустимым является предположение об изотропности отражения и рассеяния. Тогда соотношения (4) - (6) также могут быть записаны, однако ядра интегральных уравнений, записанных в терминах объемных и поверхностей плотностей радиационных потоков, содержат функции, описывающие распределение интенсивности излучения по направлениям [2]. Поэтому резольвенты в (4) - (6) зависят от $E_{T, v}$, величина $\eta_{\text {рез }}$ становится, на первый взгляд, нелинейным оператором относительно $E_{T, v}$. Однако можно показать, что и в этом случае структура оператора $\eta_{\text {рез }}$ подобна структуре соотношения (3). В качестве исходных целесообразно использовать уравнения радиационного теплообмена в терминах спектральной интенсивности эффективного излучения [4]

$$
\begin{aligned}
& I_{э^{\mathrm{TM}}, H}(N, S)=I_{-, H}(N, S)+\int_{F} r_{H}\left(N, S_{N_{1} N}\right) I_{\partial^{\mathrm{TM}}, H}\left(N_{1}, S_{N_{1} N}\right) K_{H}^{\prime}\left(N, N_{1}\right) p_{H}\left(N, S_{N_{1} N}, S\right) d F_{N_{1}}+ \\
& +\int_{V} r_{H}\left(N, S_{M_{1} N}\right) J^{\prime}{ }_{{ }^{\mathrm{TM}}, H}\left(M_{1}, S_{M_{1} N}\right) K_{H}^{\prime}\left(N, M_{1}\right) p_{H}\left(N, S_{M_{1} N}, S\right) d V_{M_{1}}, \\
& J^{\prime}{ }_{{ }^{\mathrm{TM}}, H}(M, S)=j_{-, H}(M)+B_{H}(M) \int_{F} I_{\text {эे }^{\mathrm{TM}}, H}\left(N_{1}, S_{N_{1} M}\right) K_{H}^{\prime}\left(M, N_{1}\right) \Gamma_{H}\left(M, S_{N_{1} M}, S\right) d F_{N_{1}}+ \\
& +B_{H}(M) \int_{V} J^{\prime}{ }_{\rho^{\mathrm{T}}{ }_{H}}\left(M, S_{M_{1} M}\right) K_{H}^{\prime}\left(M, M_{1}\right) \Gamma_{H}\left(M, S_{M_{1} M}, S\right) d V_{M_{1}},
\end{aligned}
$$

где $M M_{1} \in V, \quad N, N_{1} \in F ; \mathrm{S}$ - направление излучения; $r_{v}$ - отражательная способность; $\beta_{v}$ -коэффициент рассеяния; $p_{v}, \gamma_{v}$ - индикатрисы отражения и рассеяния; $I_{c, v}(N, S)=\varepsilon_{v, S}(N, S) I_{v, T}\left(T_{W}(N)\right)$ - спектральная интенсивность собственного излучения граничной поверхности ( $I_{v, T}$ - спектральная интенсивность равновесного излучения); $j_{c, v}(M)=\eta_{c, v}(M) / 4 \pi$ - спектральный коэффициент спонтанного излучения среды, $\left.\eta_{c, v}(M)=4 \alpha_{v}^{\prime}(M) E_{T, v}(T(M))\right) ; I_{э \phi, v}, J_{\text {э }, v}^{\prime}$ - спектральная интенсивность и спектральный коэффициент эффективного излучения; ядра $K_{v}^{\prime}$ - неотрицательные функции координат точек в объеме и на поверхности (структура ядер $K_{v}^{\prime}$ приведена, например, в [4]).

Уравнения (9), (10) являются интегральными уравнениями Фредгольма второго рода. Их решения могут быть формально записаны в виде

$$
\begin{aligned}
& I_{\ni \phi, v}(N, S)=\int_{F} I_{c, v}\left(N_{1}, S\right) \Gamma_{F F}^{\prime}\left(N, N_{1}, S_{N_{1} N}, S\right) d F_{N_{1}}+\int_{V} j_{c, v}(M) \Gamma_{V F}^{\prime}\left(N, M_{1}, S_{M_{1} N}, S\right) d V_{M_{1}}, \\
& j_{\ni \phi, v}^{\prime}(M, S)=\int_{F} I_{c, v}\left(N_{1}, S\right) \Gamma_{F V}^{\prime}\left(M, N_{1}, S_{N_{1} M}, S\right) d F_{N_{1}}+\int_{V} j_{c, v}(M) \Gamma_{V V}^{\prime}\left(M, M_{1}, S_{M_{1} M}, S\right) d V_{M_{1}},
\end{aligned}
$$

где $\Gamma_{F F}^{\prime}, \Gamma_{V F}^{\prime}, \Gamma_{F V}^{\prime}, \Gamma_{V V}^{\prime}$ - резольвенты ядер интегральных уравнений (9), (10).

Тогда слагаемые в правой части (1) можно записать в форме, аналогичной (4) - (6)

$$
\eta_{\text {погл }}(T)=\int_{v=0}^{\infty}\left[\alpha_{v}^{\prime}(M) \int_{V} 4 \alpha_{v}^{\prime}\left(M_{1}\right) E_{T, v}\left(T\left(M_{1}\right)\right) \widetilde{\Gamma}_{V V, v}\left(M, M_{1}\right) d V_{M_{1}}\right] d v
$$




$$
\begin{gathered}
\eta_{\text {nогл }}\left(T_{w}\right)=\int_{v=0}^{\infty}\left[\alpha_{v}^{\prime}(M) \int_{F} \varepsilon_{v}\left(N_{1}\right) E_{T, v}\left(T_{w}\left(N_{1}\right)\right) \widetilde{\Gamma}_{F V, v}\left(M, N_{1}\right) d F_{N_{1}}\right] d v, \\
\eta_{c}(T)=\int_{v=0}^{\infty}\left[4 \alpha_{v}^{\prime}(M) E_{T, v}\left(T\left(M_{1}\right)\right)\right] d v,
\end{gathered}
$$

где $\widetilde{\Gamma}_{F V, v}, \widetilde{\Gamma}_{V V, v}$ - некоторые неотрицательные функции оптико-геометрических характеристик системы.

Для построения вычислительных алгоритмов именно структура выражений (14) - (15) и знакоопределенность ядер $\widetilde{\Gamma}_{F V, v}, \widetilde{\Gamma}_{V V, v}$ являются наиболее существенными факторами.

Рассмотрим алгоритм метода итерационного расщепления. Решение задачи сложного теплообмена (1), в силу ее существенной нелинейности, может быть построено, как правило, лишь на основе использования итерационных методов. Достаточно общие итерационные методы вычислительной математики $[6,7]$ не учитывают особенностей физического содержания и математической структуры отдельных компонент операторных уравнений типа (1) и в силу этого не позволяют эффективным образом эти особенности использовать.

В данном случае необходимо отметить, что краевые задачи для дифференциальных уравнений энергии и интегральные уравнения радиационного теплообмена существенным образом отличаются как по своей структуре, так и по вычислительным алгоритмам их решения. Анализ такого рода особенностей показывает, что задачи, порожденные операторами $D$ и $\eta_{\text {погл }}-\eta_{c}$, целесообразно решать раздельно.

Кроме того необходимо отметить, что задачи радиационного теплообмена в обратной, по классификации Ю.А. Суринова, постановке не имеют единственного решения [8]. Поэтому в ходе итерационного процесса целесообразно по известному температурному полю определять радиационный тепловой поток $\eta_{\text {погл }}(T)+\eta_{\text {погл }}\left(T_{w}\right)-\eta_{c}(T)$, а затем решать краевую задачу для уравнения энергии с известной правой частью.

Этому требованию удовлетворяет итерационный процесс вида

$$
\begin{gathered}
D T_{n+1}+\omega T_{n+1}=\omega T_{n}+\eta_{\text {nогл }}\left(T_{n}\right)+\eta_{\text {nогл }}\left(T_{w}\right)-\eta_{c}\left(T_{n}\right), \\
T_{n+1 \mid \tau=0}=T^{0}, \quad T_{n+1 \mid F}=T_{w},
\end{gathered}
$$

где $\mathrm{n}$ - номер итерации; $\omega$ - итерационный параметр, наличие которого позволяет управлять сходимостью процесса. Параметр (или в общем случае, как будет показано в дальнейшем, функция) $\omega$ должен быть построен на основе априорной информации об операторах задачи.

Обычным приемом при анализе итерационных процессов является рассмотрение соответствующего итерационного процесса для задачи, линеаризованной в окрестности точного решения $[9,10]$. Используем тот же прием при построении итерационного алгоритма решения задачи (1).

Пусть начальное приближение $T_{0}$ выбрано достаточно близким к некоторому точному решению $T_{*}$ задачи (1). Линеаризуем (16) в окрестности $T_{*}$. Тогда вместо (16), (17) имеем

$$
\begin{gathered}
D T_{n+1}+\omega T_{n+1}=\omega T_{n}+\eta^{\prime}{ }_{\text {погл }}\left(T_{*}\right) T_{n}-\eta^{\prime} c\left(T_{*}\right) T_{n}+\psi, \\
T_{n+1 \mid \tau=0}=T^{0}, \quad T_{n+1 \mid F}=T_{w},
\end{gathered}
$$

где $\eta_{\text {nогл }}^{\prime}\left(T_{*}\right)$ и $\eta_{c}^{\prime}\left(T_{*}\right)$ - производные Фреше операторов $\eta_{\text {погл }}, \eta_{c}$ в точке $T_{*}$; $\psi=\eta_{\text {погл }}^{\prime}\left(T_{*}\right) T_{*}-\eta_{c}^{\prime}\left(T_{*}\right) T_{*}-\eta_{\text {погл }}\left(T_{*}\right)+\eta_{c}\left(T_{*}\right)+\eta_{\text {погл }}\left(T_{w}\right)$.

В данном случае, с учетом принятых допущений,

$$
\begin{gathered}
\eta_{\text {погл }}^{\prime}\left(T_{*}\right) T_{n}=\int_{v=0}^{\infty}\left[\alpha_{v}^{\prime}(M) \int_{V} 4 \alpha_{v}^{\prime}\left(M_{1}\right) E_{{ }_{T, v}}^{{ }^{\prime}}\left(T_{*}\left(M_{1}\right)\right) T_{n}\left(M_{1}\right) \Gamma_{V V, v}\left(M, M_{1}\right) d V_{M_{1}}\right] d v, \\
\eta_{c}^{\prime}\left(T_{*}\right) T_{n}=T_{n}(M) \int_{v=0}^{\infty}\left[4 \alpha_{v}^{\prime}(M) E_{T, v}^{\prime}\left(T_{*}(M)\right)\right] d v,
\end{gathered}
$$


где $E_{T, v}^{\prime}\left(T_{*}\right)={\frac{\partial E_{T, v}}{\partial T}}_{\mid T=T_{*}}=\frac{h v}{k T_{*}^{2}} \frac{e^{\frac{h v}{k T_{*}}}}{e^{\frac{h v}{k T_{*}}}-1} E_{0, v}\left(T_{*}\right)$ в обозначениях [4].

Введем невязку линеаризованного уравнения энергии на $\mathrm{n}$ - ной итерации

$$
Z_{n}=D T_{n}-\eta_{\text {погл }}^{\prime}\left(T_{*}\right) T_{n}+\eta_{c}^{\prime}\left(T_{*}\right) T_{n}-\phi .
$$

Пусть начальное приближение $T_{0}$ удовлетворяет краевым условиям (17). Тогда итерационный процесс (18), (19) может быть записан следующим образом:

$$
\begin{gathered}
Z_{n+1}=\omega U_{n+1}+\eta_{\text {погл }}^{\prime}\left(T_{*}\right) U_{n+1}-\eta_{c}^{\prime}\left(T_{*}\right) U_{n+1}, \\
D U_{n+1}+\omega U_{n+1}=Z_{n}, \\
U_{n+1 \mid \tau=0}=0, U_{n+1 \mid F}=0,
\end{gathered}
$$

(25)

где $U_{n+1}=T_{n}-T_{n+1}$ - поправка на (n+1)-ом шаге.

Из (24), (25) следует, что поправка $U_{n+1}$ является решением краевой задачи для уравнения энергии с однородными краевыми условиями и может быть записана в виде [11]

$$
U_{n+1}=G Z_{n}
$$

где $G$ - нелинейный интегральный оператор, ядром которого является функция Грина для задачи (24), (25).

После формальной подстановки (26) в (23) можно записать

$$
Z_{n+1}=\left(\omega I+\eta_{\text {погл }}^{\prime}\left(T_{*}\right)-\eta_{c}^{\prime}\left(T_{*}\right)\right) G Z_{n},
$$

где $I$ - единичный оператор.

Итерационный процесс (27) сходится, если в некотором нормированном пространстве $\left\|Z_{n+1}\right\| \rightarrow 0$ при $n \rightarrow \infty$. Условием сходимости является соотношение $\rho\left(\left(\omega I+\eta_{\text {погл }}^{\prime}\left(T_{*}\right)-\eta_{c}^{\prime}\left(T_{*}\right)\right) G\right)<1$, где $\rho(\bullet)$ - спектральный радиус оператора, или достаточное условие $\|\left(\omega I+\eta^{\prime}\right.$ погл $\left.\left(T_{*}\right)-\eta^{\prime}{ }_{c}\left(T_{*}\right)\right) G \|<1[10]$.

Следовательно, в общем случае параметр $\omega$ должен определяться условием минимума нормы или спектрального радиуса оператора шага итерационного процесса (27). Некоторые конкретные варианты построения величины $\omega$ на основе использования априорной информации об операторах задачи приведены в ряде работ автора.

Очевидно, что объем априорной информации об операторах в соотношении (27) ограничен, и построение параметра (в общем случае - функции) $\omega$, обеспечивающего сходимость при любых значениях параметров задачи, трудно осуществимо либо невозможно. В то же время расширение диапазона сходимости при заданном $\omega$ может быть достигнуто на основе использования идей итерационных методов вариационного типа, учитывающих апостериорную информацию об итерационном процессе. Такого рода подходы описаны, например, в [7]. Реализация методов такого типа предусматривает три основных этапа:

1) выбор минимизируемого функционала;

2) параметризация функционала;

3) минимизация функционала по выбранной системе параметров.

Рассмотрим возможный вариант решения этих вопросов применительно к рассматриваемому классу задач.

Так как единственным признаком точного решения задачи (1) является равенство нулю невязки уравнения энергии при условии удовлетворения соответствующим краевым условиям, в качестве минимизируемого функционала, характеризующего качество приближенного решения $T_{n}$, примем величину нормы невязки $\left\|Z_{n}\right\|$. Учитывая (7), будем считать, что $Z_{n} \in C_{2}(V) \times C_{2}\left(V_{\tau}\right)$. Норму и скалярное произведение в $C_{2}(V) \times C_{2}\left(V_{\tau}\right)$ введем соотношениями

$$
\|\bullet\|^{2}=\int_{V} \int_{V_{\tau}}(\bullet)^{2} d V d \tau, \quad(\bullet, \bullet)=\int_{V} \int_{V_{\tau}}(\bullet)(\bullet) d V d \tau
$$


С целью параметризации функционала $\left\|Z_{n}\right\|$ введем разбиение пространственновременной области определения задачи $V \times V_{\tau}$ на совокупность непересекающихся подобластей $\Omega_{i}$, таких, что $\bigcup_{i=1}^{N} \Omega_{i}=V \times V_{\tau}, \quad \Omega_{i} \bigcap \Omega_{j}=\varnothing, \quad(i \neq j ; i=1,2, \ldots, N ; j=1,2, \ldots N)$.

Представим невязку $Z_{n}$ в виде суммы финитных составляющих

$$
Z_{n}=\sum_{i=1}^{N} Z_{i, n}, Z_{i, n}(M, \tau)=\left\{\begin{array}{ll}
Z_{n}(M, \tau), & (M, \tau) \in \Omega_{i} \\
0, & (M, \tau) \notin \Omega_{i}
\end{array} \quad, \quad M \in V, \tau \in V_{\tau} .\right.
$$

На основе разбиения (29) поправку $U_{n+1}$ представим в виде линейной комбинации

$$
U_{n+1}=\sum_{i=1}^{N} A_{i, n+1} U_{i, n+1 / 2}
$$

где функции $U_{i, n+1 / 2}$ определяются краевыми задачами типа (24), (25):

$$
\begin{gathered}
D U_{i, n+1 / 2}+\widetilde{\infty} U_{i, n+1 / 2}=Z_{i, n} ; \\
U_{i, n+1 / 2 \mid \tau=0}=0, U_{i, n+1 / 2 \mid F}=0 .
\end{gathered}
$$

Итерационный параметр $\nsim$ представляет собой некоторое приближенное значение итерационного параметра $\omega$.

Тогда, исключая $\omega U_{n+1}$ из (23) - (25), с учетом (30) получаем

$$
Z_{n+1}=Z_{n}-\sum_{i=1}^{N} A_{i, n+1}\left(D U_{i, n+1 / 2}-\eta^{\prime}{ }_{\text {погл }}\left(T_{*}\right) U_{i, n+1 / 2}+\eta_{c}^{\prime}\left(T_{*}\right) U_{i, n+1 / 2}\right)
$$

или

$$
Z_{n+1}=Z_{n}-\sum_{i=1}^{N} A_{i, n+1}\left(Z_{i, n}-Z_{i, n+1 / 2}\right),
$$

где $Z_{i, n+1 / 2}$ представляет собой невязку линеаризованного уравнения энергии на функции $T_{i, n+1 / 2}=T_{n}-U_{i, n+1 / 2}$, т. е.

$$
Z_{i, n+1 / 2}=D T_{i, n+1 / 2}-\eta_{\text {nогл }}^{\prime}\left(T_{*}\right) T_{i, n+1 / 2}+\eta^{\ni}\left(T_{*}\right) T_{i, n+1 / 2}-\psi .
$$

Параметры $A_{i, n+1}$ в (30) определим из условия минимума нормы невязки на $(n+1)$-й итерации

$$
\left\|Z_{n+1}\right\|^{2} \underset{A_{i, n+1}}{\longrightarrow} \min .
$$

Определение параметров $A_{i, n+1}$ на основе соотношений (28), (32), (33) с использованием условия $\partial\left\|Z_{n+1}\right\|^{2} / \partial A_{i<n+1}=0$ приводит к системе линейных алгебраических уравнений вида

$$
\mathbf{W}(\mathbf{Z}) \mathbf{A}=\Psi(\mathbf{Z})
$$

где обозначено $\mathbf{A}=\left[A_{1, n+1} A_{2, n+1} \ldots A_{N, n+1}\right]^{T}, \mathbf{W}$ - квадратная матрица $\mathrm{NxN}$ с элементами $W(Z)_{i, j}=\left(Z_{i, n}-Z_{i, n+1 / 2}, Z_{j, n}-Z_{j, n+1 / 2}\right)$, матрица-столбец $\Psi(\mathbf{Z})$ содержит скалярные произведения вида $\Psi(Z)_{i}=\left(Z_{n}, Z_{i, n}-Z_{i, n+1 / 2}\right)$. Выбранный способ параметризации невязки с помощью разбиения (29) гарантирует линейную независимость строк матрицы $\mathbf{W}$ и, следовательно, существование единственного решения системы уравнений (35) [12].

Таким образом, предложенная схема перехода от $n$-ного к $(n+1)$-му приближению при решении задачи сложного теплообмена, линеаризованной в окрестности некоторого точного решения $T_{*}$, содержит следующие последовательно реализуемые этапы:

1) путем решения задачи радиационного теплообмена определяется поле плотности потока результирующего излучения - величина $\eta_{\text {nогл. }}^{\prime}\left(T_{*}\right) T_{n}-\eta_{-}^{\prime}\left(T_{*}\right) T_{n}+\psi$;

2) по соотношению (22) строится невязка $Z_{n}$;

3) строится разбиение (29); 
4) решаются краевые задачи (31) относительно $U_{i, n+1 / 2}$;

5) строятся функции $T_{i, n+1 / 2}$;

6) решается задача радиационного теплообмена для определения плотности потока результирующего излучения $\eta_{\text {погл }}^{\prime}\left(T_{*}\right) T_{i, n+1 / 2}-\eta_{c}^{\prime}\left(T_{*}\right) T_{i, n+1 / 2}+\psi$;

7) по соотношению (33) строятся невязки $Z_{i, n+1 / 2}$;

8) формируются матрицы $\mathbf{W}$ и $\Psi(\mathbf{Z})$ и решается система линейных алгебраических уравнений (35) относительно $\mathbf{A}$;

9) строится следующее приближение по температурному полю

$$
T_{n+1}=T_{n}-\sum_{i=1}^{N} A_{i, n+1} U_{i, n+1 / 2} .
$$

Сходимость предложенного итерационного процесса для линеаризованной задачи при достаточно точном нулевом приближении гарантирует сходимость соответствующего итерационного процесса для нелинейной задачи (1) при $T_{*}=T_{n}[10]$.

Таким образом, в структуре итерационного процесса априорная информация, выраженная в функции $\widetilde{\varpi}$, учитывается при формировании поправок $U_{i, n+1 / 2}$, апостериорная - при вычислении параметров минимизации нормы невязки $A_{i, n+1}$.

Необходимо также отметить, что при реализации сформулированного алгоритма решение задачи сложного теплообмена распадается на следующие основные этапы:

1) решение задач радиационного теплообмена;

2) решение краевых задач для уравнений энергии;

3) вычисление невязок уравнений энергии;

4) вычисление норм и скалярных произведений;

5) решение системы линейных алгебраических уравнений.

Возможны следующие частные случаи сформулированного выше алгоритма 1 -9:

1) если параметры $A_{i, n+1}$ не вычисляются $\left(A_{i, n+1}=1\right)$, то итерационный процесс становится стационарным и его сходимость определяется параметром (функцией) $\varpi$;

2 ) если норма невязки $Z_{n+1}$ минимизируется по одному параметру, а $\widetilde{\sigma}=0$, то итерационный процесс (29) - (36) представляет собой вариант метода минимальных невязок [6, 7]; итерационный процесс в этом случае может быть записан в канонической форме двухслойной итерационной схемы (в обозначениях [7]) $B\left(\frac{T_{n+1}-T_{n}}{\tau_{n+1}}\right)+A T_{n}=\Psi$, где в данном случае $B=D$, $A=D-\eta_{\text {погл }}^{\prime}\left(T_{*}\right)+\eta^{\prime}\left(T_{*}\right), \tau_{n+1}=A_{n+1}$.

В общем случае предложенный итерационный процесс не сводится к описанным в [7] итерационным схемам. Отметим, что сходимость итерационного процесса (29) - (36) зависит от выбора параметра (функции) $\approx$ и разбиения (29).

Определим условия сходимости описанного выше итерационного процесса. Прежде чем перейти к рассмотрению достаточно общего случая и формулировке рекомендаций по выбору ळ и построению разбиения (29), приведем некоторые предварительные соображения.

Пусть, например, $N=1$. Тогда вместо (32) - (35) получаем

$$
\begin{gathered}
Z_{n+1}=Z_{n}-A_{n+1}\left(Z_{n}-Z_{n+1 / 2}\right)=Z_{n}-A_{n+1} W U_{n+1 / 2}, \\
A_{n+1}=\frac{\left(Z_{n}, Z_{n}-Z_{n+1 / 2}\right)}{\left\|Z_{n}-Z_{n+1 / 2}\right\|^{2}}=\frac{\left(Z_{n}, W U_{n+1 / 2}\right)}{\left\|W U_{n+1 / 2}\right\|^{2}},
\end{gathered}
$$

где обозначено $W U_{n+1 / 2}=D U_{n+1 / 2}-\eta_{\text {погл }}^{\prime}\left(T_{*}\right) U_{n+1 / 2}+\eta_{c}^{\prime}\left(T_{*}\right) U_{n+1 / 2}$.

Очевидным требованием, предъявляемым к поправке $U_{n+1 / 2}$, является $\left\|W U_{n+1 / 2}\right\|^{2} \neq 0$, что выполняется, если $U_{n+1 / 2}$ не является решением однородной линеаризованной задачи теплооб- 
мена типа (18), (19). Принятый способ построения поправок путем решения краевых задач (31) обеспечивает выполнение этого условия.

Отметим, что монотонное убывание норм невязок

$$
\left\|Z_{n+1}\right\|<\left\|Z_{n}\right\|
$$

является достаточным условием фактор-сходимости рассматриваемого итерационного процесса [13]. Однако в данном случае, видимо, можно считать, что линеаризованная задача теплообмена типа (18), (19) имеет единственное решение. Поэтому в пространстве функций, удовлетворяющих однородным краевым условиям, ядро оператора $W(\operatorname{ker} W)$ содержит только нулевой элемент $(\operatorname{ker} W=0)$, и неравенство (38) является условием сходимости итерационного процесса в обычном смысле. Для выполнения этого условия достаточно гарантировать $A_{n+1}>0$.

Соотношение величин радиационных и конвективно-кондуктивных тепловых потоков в задачах сложного теплообмена может быть различным, и, в том числе, таким, которое приводит к малым величинам $A_{n+1}$ или, в каких-то случаях, к $A_{n+1}=0$, что влечет за собой соответственно медленную сходимость или "застревание" итерационного процесса. При этом достаточно высокая скорость сходимости может быть достигнута лишь при определенных значениях свободных параметров итерационного процесса.

Построим достаточные условия сходимости применительно к двум основным классам задач сложного теплообмена.

1. Пусть задача сложного теплообмена (1) является нестационарной.

В этом случае итерационный процесс (29) - (36) сходится, если рассматриваемый временной интервал $V_{\tau}$ достаточно мал. При этом минимизация нормы невязки может быть проведена по одному параметру.

Действительно, пусть $\mathrm{N}=1$. Тогда, в соответствии с (37), можно записать

$$
A_{n+1}=\frac{\left(Z_{n}, Z_{n}-Z_{n+1 / 2}\right)}{\left\|Z_{n}-Z_{n+1 / 2}\right\|^{2}}=\frac{\left\|Z_{n}\right\|^{2}}{\left\|Z_{n}-Z_{n+1 / 2}\right\|^{2}}\left(1-\frac{\left(Z_{n}, Z_{n+1 / 2}\right)}{\left\|Z_{n}\right\|^{2}}\right)
$$

Аналогично соотношениям (26), (27), исходя из (31), можно записать $U_{n+1 / 2}=G Z_{n}$, $Z_{n+1}=\left(ळ I+\eta_{\text {nогл }}^{\prime}\left(T_{*}\right)-\eta_{c}^{\prime}\left(T_{*}\right)\right) G Z_{n}$.

Используя неравенство Коши-Буняковского, получаем оценку

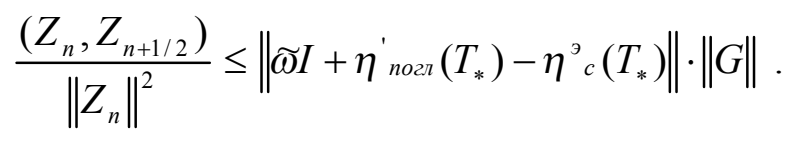

Учитывая, что $\eta_{c}^{\prime}\left(T_{*}\right)$ является функцией вида (21), зададим параметр ळ следующим образом:

$$
\varpi=\eta_{c}^{\prime}\left(T_{*}\right)=\int_{v=0}^{\infty}\left[4 \alpha_{v}^{\prime}(M) E_{T, v}^{\prime}\left(T_{*}(M)\right)\right] d v .
$$

Тогда, используя (21) и оценку нормы интегрального оператора, приведенную в [9], запишем

$$
\begin{gathered}
\left\|\widetilde{\varpi} I+\eta_{\text {погл }}^{\prime}\left(T_{*}\right)-\eta^{\prime}{ }_{c}\left(T_{*}\right)\right\|=\left\|\eta_{\text {погл }}^{\prime}\left(T_{*}\right)\right\| \leq \\
\leq\left[\iint_{V} \int_{V_{\tau}} \mid \int_{V=0}^{\infty} 4\left[\alpha_{v}^{\prime}(M) \alpha_{v}^{\prime}\left(M_{1}\right) E_{T, v}^{\prime}\left(T_{*}\left(M_{1}\right)\right) \Gamma_{V V, v}\left(M, M_{1}\right) d v\right]^{2} d V_{M_{1}} d V_{M} d \tau\right]^{1 / 2} .
\end{gathered}
$$

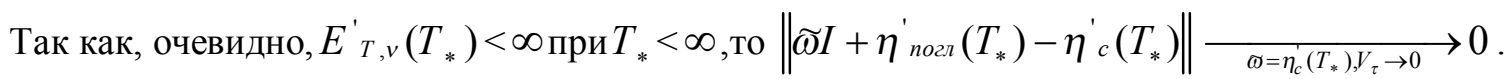


Оператор Грина $\mathrm{G}$ является непрерывным и ограниченным в $L_{2}[11]$, т. е. $\|G\|<\infty$. Поэтому, исходя из приведенных выше оценок, получаем $\frac{\left(Z_{n}, Z_{n+1 / 2}\right)}{\left\|Z_{n}\right\|^{2}} \underset{V_{\tau} \rightarrow 0}{\longrightarrow} 0$.

Следовательно, при достаточно малой величине временного интервала $V_{\tau}$ выполняется неравенство $\frac{\left(Z_{n}, Z_{n+1 / 2}\right)}{\left\|Z_{n}\right\|^{2}}<1$.

Учитывая, что множитель перед скобкой в (31) очевидно положителен, при достаточно малом $V_{\tau}$, получаем $A_{n+1}>0$, что и гарантирует убывание нормы невязки и, следовательно, сходимость итерационного процесса.

2. Пусть задача сложного теплообмена является стационарной.

В этом случае норма и скалярное произведение определяются соотношениями $\|\bullet\|^{2}=\int_{V}(\bullet)^{2} d V$, $(\bullet, \bullet)=\int_{V}(\bullet) \cdot(\bullet) d V$. Для выполнения условия сходимости (38) достаточно наличия хотя бы одного ненулевого параметра $A_{i, n+1}$. Это означает, что система уравнений (35) должна иметь нетривиальное решение. Данное требование выполняется, если хотя бы один элемент матрицы $\Psi(Z)$ не равен нулю, что обеспечивается, по крайней мере, при специальном выборе параметра ळ и разбиения (29).

Пусть, аналогично (41),

$$
\widetilde{\varpi}=\eta_{c}^{\prime}\left(T_{*}\right)=\int_{v=0}^{\infty} 4 \alpha_{v}^{\prime}(M) E_{T, v}^{\prime}\left(T_{*}(M)\right) d v .
$$

Построим разбиение (29) в виде $Z_{i, n}(M)=\left\{\begin{array}{ll}Z_{n}(M), & (M, \tau) \in V_{i} \\ 0, & (M, \tau) \notin V_{i}\end{array}\right.$, выделив достаточно малую подобласть $V_{j} \subset V$, в которой $\left|Z_{n}\right|$ достигает наибольшего значения, а сама невязка является знакопостоянной.

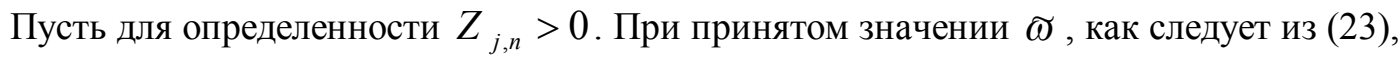

$$
Z_{j, n+1 / 2}=\eta_{\text {nогл }}^{\prime}\left(T_{*}\right) U_{j, n+1 / 2}
$$

В краевой задаче (31) величина $Z_{i, n}$ представляет собой объемный тепловой источник. Свойства дифференциального оператора уравнения энергии, очевидно, таковы, что знак $U_{i, n+1 / 2}$ совпадает со знаком $Z_{i, n}$, если $Z_{i, n}$ - знакопостоянная функция. Следовательно, в данном случае $U_{i, n+1 / 2} \geq 0$. Тогда, так как $E_{T, v}^{\prime}>0$ и $\Gamma_{V V, v} \geq 0$, из соотношения (20) следует, что $Z_{j, n+1 / 2}=\eta_{\text {погл }}^{\prime}\left(T_{*}\right) U_{j, n+1 / 2} \geq 0$.

Рассмотрим ј-тый элемент матрицы $\Psi(Z)$. Так как $Z_{n}$ - непрерывная функция, на основании теоремы о среднем запишем

$$
\left(Z_{n}, Z_{j, n}-Z_{j, n+1 / 2}\right)=Z_{n}^{j} \int_{V_{j}} Z_{j, n} d V-Z_{n} \int_{V} Z_{j, n+1 / 2} d V
$$

где $Z_{n}^{j}$ и $Z_{n}$ - некоторые средние значения $Z_{n}$ в областях $V_{j}$ и $V$ соответственно.

Оценим интегралы в (44), используя соотношения, учитывающие особенности рассматриваемой задачи теплообмена. Из соотношения $U_{i, n+1 / 2} \geq 0$ с учетом однородного граничного условия в (31) $\left(U_{i, n+1 / 2 \mid F}=0\right)$ следует оценка

$$
\int_{V} D U_{j, n+1 / 2} d V>0
$$

Действительно, в данном случае 


$$
\int_{V} D U_{j, n+1 / 2} d V=\int_{V} \operatorname{div}\left(c_{p} \rho \vec{v} U_{j, n+1 / 2}\right) d V-\int_{V} \operatorname{div}\left(\lambda \nabla U_{j, n+1 / 2}\right) d V .
$$

Используя теорему Гаусса-Остроградского, получаем

$$
\int_{V} D U_{j, n+1 / 2} d V=\int_{F} c_{p} \rho v U_{j, n+1 / 2} \vec{v} \cdot \vec{n} d F-\int_{F} \lambda \nabla U_{j, n+1 / 2} \vec{n} d F,
$$

где $\vec{n}$ - внешняя нормаль к поверхности $F$. Учитывая далее приведенные выше свойства функции $U_{i, n+1 / 2}$, имеем

$$
\int_{F} c_{p} \rho v U_{j, n+1 / 2} \vec{v} \cdot \vec{n} d F=0, \int_{F} \lambda \nabla U_{j, n+1 / 2} \vec{n} d F<0 .
$$

Отсюда и следует оценка (45).

Второе соотношение связано с оценкой радиационных тепловых потоков. Тепловой поток $\eta_{\text {погл }}^{\prime}\left(T_{*}\right) U_{j, n+1 / 2}$ определяется путем решения задачи радиационного теплообмена в фундаментальной постановке при $E_{T, v}\left(T_{W}\right)=0, E_{T, v}(T)=\eta_{c}^{\prime}\left(T_{*}\right) U_{j, n+1 / 2}$. Так как $U_{i, n+1 / 2} \geq 0$ и $\eta_{c}^{\prime}\left(T_{*}\right)>0$, плотность потока равновесного излучения $E_{T, v}(T) \geq 0$ для всех точек в объеме $V$. Плотность потока равновесного излучения ограничивающих поверхностей $E_{T, v}\left(T_{W}\right)=0$. В этих условиях, очевидно, объем $V$ теряет теплоту излучением, т.е. $\int_{V} \eta_{\text {рез }} d V<0$. Так как, по определению [4], $\eta_{\text {рез }}=\eta_{\text {погл }}-\eta_{c}$, то в рассматриваемом случае

$$
\int_{V} \eta_{\text {pes }} d V=\int_{V} \eta_{\text {nогл }}^{\prime}\left(T_{*}\right) U_{j, n+1 / 2} d V-\int_{V} \eta_{c}^{\prime}\left(T_{*}\right) U_{j, n+1 / 2} d V<0 .
$$

Отсюда и следует искомая оценка

$$
\int_{V} \eta_{\text {nогл }}^{\prime}\left(T_{*}\right) U_{j, n+1 / 2} d V<\int_{V} \eta_{c}^{\prime}\left(T_{*}\right) U_{j, n+1 / 2} d V .
$$

Тогда из уравнения (31) с учетом соотношений (42), (43) и оценок (45), (46) последовательно получаем

$$
\begin{aligned}
& \int_{V_{j}} Z_{j, n} d V=\int_{V} Z_{j, n} d V=\int_{V} D U_{j, n+1 / 2} d V+\int_{V} \widetilde{\omega} U_{j, n+1 / 2} d V=\int_{V} D U_{j, n+1 / 2} d V+\int_{V} \eta_{-}^{\mathbb{O}}\left(T_{*}\right) U_{j, n+1 / 2} d V> \\
& >\int_{V} \eta_{-}^{\odot}\left(T_{*}\right) U_{j, n+1 / 2} d V>\int_{V} \eta_{\cdot{ }^{\circ}, \sqrt{b}}\left(T_{*}\right) U_{j, n+1 / 2} d V=\int_{V} Z_{j, n+1 / 2} d V \\
& \text { т. е. } \\
& \qquad \int_{V} Z_{j, n} d V>\int_{V} Z_{j, n+1 / 2} d V .
\end{aligned}
$$

Кроме того, выбранный способ выделения подобласти $V_{j} \subset V$ гарантирует выполнение неравенства

$$
Z_{n}^{j}>Z_{n}
$$

при достаточно малом объеме области $V_{j}$.

Используя неравенства (47), (48), из (44) получаем оценку

$$
\left(Z_{n}, Z_{j, n}-Z_{j, n+1 / 2}\right)>0 \text {, }
$$

что и гарантирует существование ненулевого элемента в ј-той строке матрицы $\Psi(Z)$ и выполнение условия сходимости (38).

Если $Z_{j, n}<0$ в области $V_{j}$, то, очевидно, $U_{i, n+1 / 2} \leq 0, Z_{j, n+1 / 2} \leq 0$, знаки неравенств в (45), (47), (48) изменяются на противоположные. Тогда вместо (49) получаем неравенство $\left(Z_{n}, Z_{j, n}-Z_{j, n+1 / 2}\right)<0$, что также гарантирует выполнение условия сходимости (38).

Таким образом, итерационный процесс (29), (31) - (36), основанный на процедуре минимизации нормы невязки линеаризованного уравнения энергии по системе параметров, сходится по квадратичной норме, если выполняются следующие условия. 


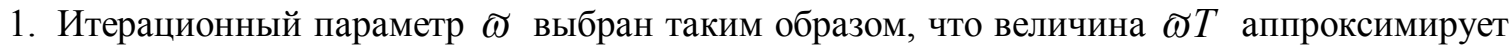
в линеаризованной задаче величину собственного излучения элементов объема, т.е.

$$
\varpi=\eta_{c}^{\prime}\left(T_{*}\right)=\int_{v=0}^{\infty} 4 \alpha_{v}^{\prime}(M) E_{T, v}^{\prime}\left(T_{*}(M)\right) d v .
$$

2. В нестационарных задачах рас-

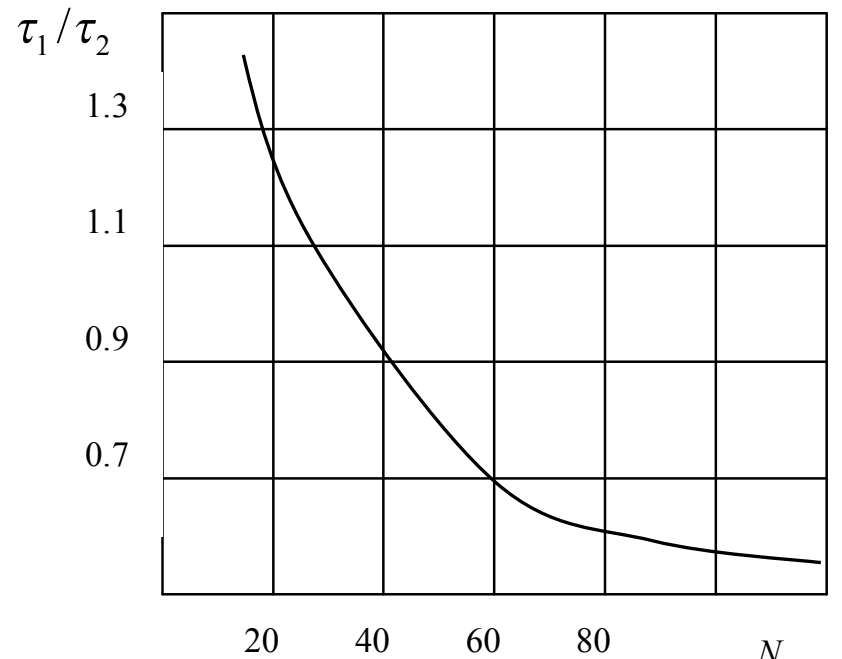

Влияние количества зон в расчетной сетке задачи радиационного теплообмена на относительный объем вычислительных затрат четный интервал времени $V_{\tau}$ достаточно мал.

3. В стационарных задачах разбиение области V на подобласти построено таким образом, что в некоторой подобласти $V_{j}$ содержится $\max _{V}\left|Z_{n}\right| ;$ невязка $Z_{n}$ в этой подобласти является знакопостоянной величиной.

Условие 3 гарантирует сходимость итерационного процесса при наличии по крайней мере двух параметров минимизации $\left\|Z_{n+1}\right\|$.

Если начальное приближение $T_{0}$ задано достаточно близким к точному решению $T_{*}$, то сходимость итерационного процесса для линеаризованной задачи гарантирует сходимость аналогичного итерационного процесса для исходной нелинейной задачи сложного теплообмена [10].

С целью оценки эффективности метода итерационного расщепления проведено его сопоставление с известным зонально-узловым методом решения задач данного класса [14]. В качестве тестовой решалась задача сложного теплообмена при гидродинамически стабилизированном ламинарном течении в канале на основе "серой" модели излучения. При этом использовалась однопараметрическая схема минимизации нормы невязки (количество параметров минимизации $N=1$ ). Алгоритмы зонально-узлового метода и метода итерационного расщепления реализовывались на основе одних и тех же базовых модулей при одинаковой конечномерной аппроксимации радиационной части задачи. Количество зон разбиения излучающего объема варьировалось. При реализации метода итерационного расщепления для решения конвективнокондуктивной части задачи использовался алгоритм прогонки, для решения радиационной части задачи - итерационный метод Зейделя. Для сопоставления эффективности методов фиксировались затраты машинного времени. Результаты сопоставления указанных методов приведены на рисунке, где $\tau_{1}$ и $\tau_{1}$ - затраты машинного времени для метода итерационного расщепления и зонально-узлового метода соответственно, $N$ - количество зон разбиения излучающего объема.

Приведенные результаты показывают, что алгоритм итерационного расщепления, благодаря оптимизации его структуры и возможности использования эффективных специализированных методов решения краевых задач для уравнений энергии и интегральных уравнений радиационного теплообмена, позволяет существенно снизить объем вычислительных затрат в том случае, когда радиационный теплоперенос играет существенную роль.

Таким образом, в данной работе рассмотрены основные особенности постановки задач сложного теплообмена, существенные для анализа вычислительных алгоритмов; изложена схема метода итерационного расщепления, позволяющая обеспечить гибкую модульную структуру вычислительного алгоритма и возможность использования эффективных специализированных методов решения частных задач радиационного и конвективно-кондуктивного теплопереноса; построены достаточные условия сходимости метода итерационного расщепления; эффективность метода проиллюстрирована путем сопоставления с зонально-узловым методом. 


\section{БИБЛИОГРАФИЧЕСКИЙ СПИСОК}

1. Арончик Г.И. Декомпозиция в задачах сложного теплообмена // ИФЖ. 1989. № 5. С. 861-862.

2. Арончик Г.И. Метод итерационного расщепления в задачах сложного теплообмена. // Тепломассообмен-ММФ92. Радиационный и комбинированный теплообмен. Т. 2 . Минск: АНТ "ИТМО им. А.В. Лыкова", АНБ. 1992. C. 96-99.

3. Лыков А.В. Тепломассобмен: Справочник. М.: Энергия, 1978. 480 с.

4. Адрианов В.Н. Основы радиационного и сложного теплообмена. М.: Энергия, 1972. 464 с.

5. Смирнов В.И. Курс высшей математики. Т. 4, ч. 1. М.: Наука, 1974. 336 с.

6. Марчук Г.И. Методы вычислительной математики. М.: Наука, 1980. $536 \mathrm{c.}$

7. Самарский А.А., Николаев Е.С. Методы решения сеточных уравнений. М.: Наука, 1978. 590 с.

8. Суринов Ю.А. К теории переноса излучения и лучистого теплообмена в поглощающей и рассеивающей среде.// Изв. АН СССР. Энергетика и транспорт. 1966. N 6. С. 127-153.

9. Канторович Л.В., Акилов Г.П. Функциональный анализ. М.: Наука, 1977. 741 с.

10. Красносельский М.А., Вайникко Г.М. и др. Приближённое решение операторных уравнений. М.: Наука, 1969. $456 \mathrm{c}$.

11. Мизохата C. Теория уравнений с частными производными. М.: Мир, 1977. 504 с.

12. Воеводин В.В., Кузнецев Ю.А. Матрицы и вычисления. М.: Наука, 1984. 320 с.

13. Марчук Г.И., Кузнецов Ю.А. Итерационные методы и квадратичные функционалы //Методы вычислительной математики. Новосибирск: Наука, 1975. С. 4-143

14. Маликов Г.К., Лисиенко В.Г., Маликов Ю.К., Двинянинов А.Б. Зонально-узловой метод совместного решения уравнений гидродинамики и теплообмена излучением //ТВТ. 1985. Т. 23, N 6. С. 1103-1111. 